\title{
Detwinning-induced reduction in ductility of twinned copper nanowires
}

\author{
ZHANG JunJie ${ }^{1,2 *}$ XU FangDa ${ }^{1}$, YAN YongDa ${ }^{1,3} \&$ SUN Tao $^{1}$ \\ ${ }^{1}$ Center for Precision Engineering, Harbin Institute of Technology, Harbin 150001, China; \\ ${ }^{2}$ Interdisciplinary Centre for Advanced Materials Simulation (ICAMS), Ruhr University Bochum, Universitätsstr. $90 a$, D-44801 Bochum, Germany; \\ ${ }^{3}$ Key Laboratory of Micro-systems and Micro-structures Manufacturing of Ministry of Education, Harbin Institute of Technology, Harbin \\ 150001, China
}

Received July 13, 2012; accepted September 20, 2012; published online December 11, 2012

\begin{abstract}
Detwinning is a unique deformation mechanism of nanotwinned metals with twin lamellae thickness down to a few nanometers. In this work we investigate the impact of detwinning mechanism on the tensile ductility of twinned Cu nanowires containing high density of parallel twin boundaries by means of molecular dynamics simulations. Simulation results show that the fracture strain of twinned $\mathrm{Cu}$ nanowires has a strong dependence on twin boundary spacing, resulting from the competition between individual deformation modes. Particularly for the twinned $\mathrm{Cu}$ nanowires containing the thinnest twin lamellaes, the dominant detwinning mechanism leads to a significant reduction in the tensile ductility. It is found that detwinning originates from twin boundary migration, which is a result of the glide of lattice partial dislocations on the twin planes. This work advances our fundamental understanding of the twin boundary-related mechanical properties of twinned metallic nanowires.
\end{abstract}

Cu nanowires, twin boundary, uniaxial tension, detwinning, molecular dynamics

Citation: Zhang J J, Xu F D, Yan Y D, et al. Detwinning-induced reduction in ductility of twinned copper nanowires. Chin Sci Bull, 2013, 58: 684-688, doi: $10.1007 / \mathrm{s} 11434-012-5575-3$

Coherent twin boundary (TB) is a special kind of planar defects whose lattice structures exhibit mirror symmetry across the boundary. TB has drawn wide range of interest for its unique properties: it acts as effective obstacle to dislocation motion due to its low excess energy and thus strengthens materials, and has low electrical resistivity because of the coherent characteristic. Consequently, a superior combination of ultrahigh strength and high electrical conductivity was achieved in ultrafine-grained polycrystalline $\mathrm{Cu}$ by introducing high density of growth TBs $[1,2]$. Most importantly, unlike the conventional grain boundary (GB) strengthening which makes materials strong but brittle, the TB strengthening found in metallic materials is accompanied with no significant reduction in ductility, which thus provides novel possibilities to develop advanced functional nanostructures $[3,4]$. However, the role of TBs playing in

*Corresponding author (email: zhjj505@gmail.com) the ductility of metallic materials of extremely small dimensions, e.g. nanowires (NWs) with high surface to volume ratio, is distinctly different from their bulk counterparts, as the introduction of TBs does not always monotonically contribute to their ductility. Atomistic simulations have demonstrated that the introduction of fivefold growth TBs can compromise the ductility of metallic NWs significantly [5-7]. Sansoz et al. [8] found via atomistic simulations that the failure strain of $\mathrm{Au}$ NWs containing embedded TBs is significantly smaller than the single crystal one. More recent tensile experiment discovered the mechanical formed TBsinduced brittle-like fracture in sub-20 nm Au NWs [9]. Therefore, our fundamental understanding of the TBs-related ductility in metallic NWs is far from being completed.

The unique dislocation-TB interaction, as the central issue governing the mechanical properties of nanostructured materials containing $\mathrm{TBs}$, has strong dependence on twin lamellae thickness. Both experiments and atomistic simula- 
tions have demonstrated that there exists a critical twin boundary spacing (TBS) below which softening occurs. The softening is ascribed to the transition in yielding mechanism from the slip transfer across TBs to the mobilization and multiplication of pre-existing dislocations or the nucleation and motion of partial dislocations parallel to twin planes $[10,11]$. The alternation of dominant deformation mechanism in softening regime accompanied with the transition in yielding mechanism is expected to have strong impact on the ductility of materials. Particularly for metallic NWs containing high density of TBs, the TB-associated mechanism such as TB migration and detwinning may play pronounced even dominant role in the plastic deformation, because the nucleation and motion of lattice dislocations are severely restricted. In this work, we perform molecular dynamics (MD) simulations to elucidate the fundamental deformation mechanisms operating in twinned $\mathrm{Cu}$ NWs containing high density of parallel TBs subjected to uniaxial tension. We further study the influence of TBS on the deformation behavior of the NWs. We find that the dominant detwinning mechanism leads to a significant reduction in the tensile ductility of twinned $\mathrm{Cu}$ NWs with the thinnest twin lamellae thickness.

\section{Simulation method}

Figure 1 shows that four [111]-oriented $\mathrm{Cu}$ NWs, as one

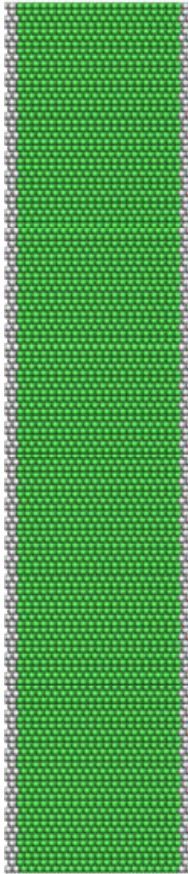

(a)

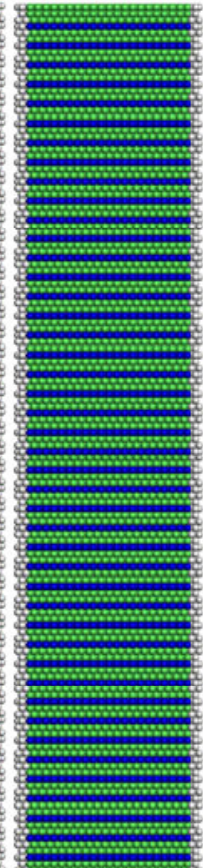

(b)

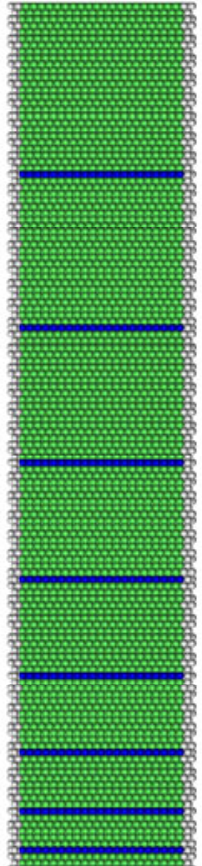

(c)

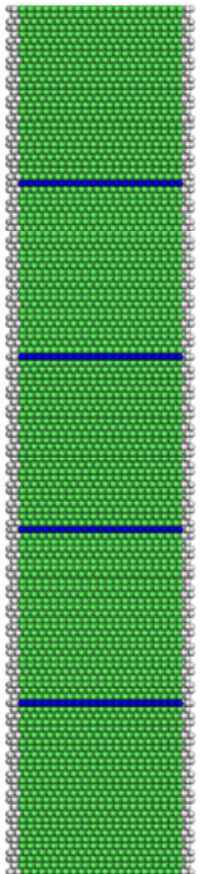

(d)
Figure 1 (Color online) Cross-sectional views of MD models of $\mathrm{Cu}$ NWs. (a) Single; (b) NW1; (c) NW19; (d) NW9. Atoms are colored according to calculated CNA values, as green for FCC atoms, blue for TB atoms, and gray for surface atoms. single crystalline and three twinned, are considered in current work. The diameter and length of those NWs with circular cross-section are the same as 5.9 and $28.2 \mathrm{~nm}$, respectively. The atomic interactions between approximately 65600 facecentered cubic (FCC) $\mathrm{Cu}$ atoms are described by the wellestablished EAM potential developed for $\mathrm{Cu}$ [12]. The three twinned $\mathrm{Cu}$ NWs have different twin lamellae thicknesses: NW1 and NW9 have monotonic TBS of 0.63 and $5.64 \mathrm{~nm}$, respectively, and NW19 has gradient TBS at a range of $0.63-5.64 \mathrm{~nm}$. Periodic boundary condition (PBC) is only applied along the [111] axial direction of the NWs, and the other directions are free. Note that, the applying of PBC along axial direction yields an infinite long length, which may result in the Rayleigh instability of NWs under high strain rate. The size-dependent deformation behavior of NWs with finite length can be found elsewhere [13,14].

Prior to uniaxial tension, the as-created NWs are subjected to following relaxation procedures to reach their equilibrium configurations: the atoms are first relaxed to their local minimum energy configurations, followed by dynamic relaxation at $30 \mathrm{~K}$ under 0 bar using the NoseHoover thermostat for $150 \mathrm{ps}$ in the isothermal-isobaric NPT ensemble. After the completion of relaxation, uniaxial tension of $\mathrm{Cu}$ NWs is performed in the canonical NVT ensemble at $30 \mathrm{~K}$ by straining the simulation box along [111] axial direction with constant strain rate of $5 \times 10^{7} \mathrm{~s}^{-1}$. The common neighbor analysis (CNA) is adopted to identify defects generated in the NWs during deformation process, and the difference between intrinsic stacking fault (ISF) and TB is further distinguished $[15,16]$. The coloring scheme is: green stands for FCC atoms, red for ISF atoms, blue for TB atoms, and gray for Other atoms including free surface and dislocation cores. All the MD simulations are performed by using the LAMMPS code with a time step of 1 fs [17]. And the OVITO is utilized to visualize MD data and generate MD snapshots [18].

\section{Results and discussion}

Figure 2 plots the stress-strain curves of the four $\mathrm{Cu}$ NWs subjected to uniaxial tension. The elastic deformation behaviors of all the NWs are approximately the same, as the stress increases linearly with the same slope until the strain reaches its elastic limit. It indicates that the Young's modulus of $\mathrm{Cu}$ NWs is not influenced by the introduction of aligned TBs, which agrees well with previous observations [13]. The calculated Young's modulus of the [111]-oriented $\mathrm{Cu}$ NWs is $161 \mathrm{GPa}$. The yield stress and associated elastic strain limit are approximately the same for all the NWs, indicating there is no obvious strengthening effect of TBs in the NWs. It should be noted that our observation is of considerable difference from the strengthening effect of TBs reported by previous studies $[19,20]$. This discrepancy may be related to the low temperature of $30 \mathrm{~K}$ and the Foiles 


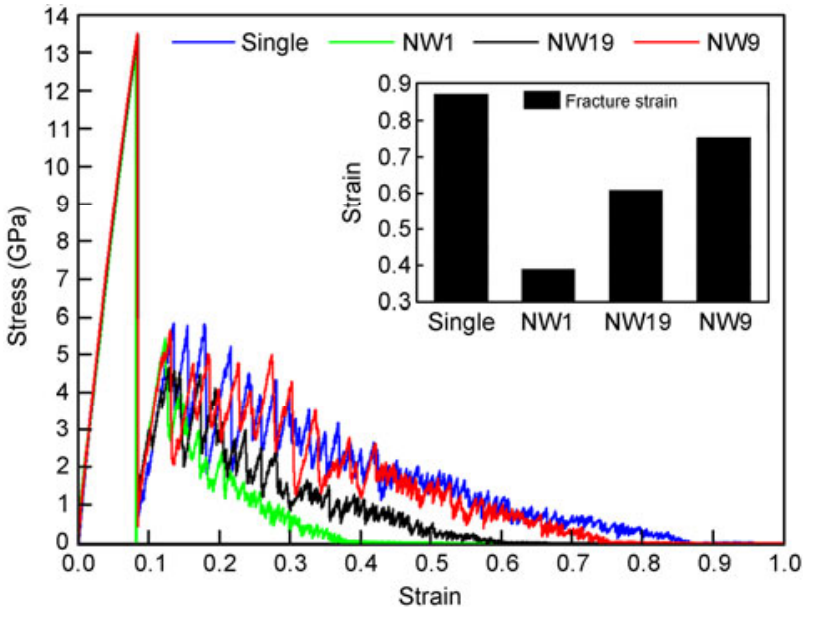

Figure 2 Stress-strain curves of uniaxial tension of $\mathrm{Cu}$ NWs. The inset shows the fracture strains of simulated NWs.

EAM potential utilized in current work. The temperaturedependent mechanical properties of NWs have been widely reported [21,22]. Furthermore, recent experimental study also suggested that the strengthening effect of TBs depends strongly on temperature [23].

It is seen from Figure 2 that the slope of dramatically decreased stress after the yield point, caused by the initiation of plasticity, is also the same for each NWs. However, there is dramatic difference in the yielding mechanism of $\mathrm{Cu}$ NWs with different twin thicknesses. The incipient plasticity of single crystal and twinned NWs with large TBS, i.e. NW19 and NW9, is governed by the nucleation of lattice partial dislocations from free surface and subsequent glide. In contrast, the yielding of NW1 is dominated by the nucleation of twinning partials from perfect TBs, which is also seen in indentation test [24]. Figure 2 demonstrates that the stress-strain curves show significant deviations after the precipitous drop of stresses. In particular, the inset of Figure 2 shows that there is distinct difference in the fracture strain of $\mathrm{Cu}$ NWs containing different internal microstructures. It is seen that the single crystalline $\mathrm{Cu}$ NWs possesses larger fracture strain than twinned ones, indicating that the tensile ductility of twinned $\mathrm{Cu}$ NWs is compromised by the presence of TBs due to the plastic localization [8]. While the lower and upper bounds of the TBS of NW19 correspond to the TBS of NW1 and NW9, respectively, it is interesting to note that the fracture strain of NW19 with gradient TBS is also intermediate among twinned $\mathrm{Cu}$ NWs. Thus, Figure 2 clearly demonstrates the twin lamellae thickness dependence of the tensile ductility of twinned $\mathrm{Cu}$ NWs: the smaller the TBS, the lower the tensile ductility.

To reveal the origin of the twin lamellae thickness-dependent tensile ductility in twinned $\mathrm{Cu}$ NWs shown in Figure 2, the deformation mechanisms of $\mathrm{Cu}$ NWs are investigated by statistically analyzing the evolutions of atoms in different lattice structures. Figure 3(a) and (b) shows the variations in the reduced numbers of FCC atoms and Other atoms upon strain, respectively. The reduced number is calculated by subtracting the base number before deformation using the measured number. For single crystalline $\mathrm{Cu}$ NWs and twinned ones with large TBS, i.e. NW19 and NW9, the number of FCC atoms decreases accompanied with increasing Other atoms prior to fracture, demonstrating that dislocation activity is the major plastic deformation mode as the change in Other atoms is mainly contributed by nucleation and motion of lattice dislocations. However, the strong fluctuations in their evolutions of FCC atoms suggest that there is another deformation mode of deformation twinning also playing role in the plastic deformation. Dynamic analysis of instantaneous defect evolutions reveals that the newly formed mechanical TB is originated from the dissociation of lattice partial dislocations, which consequently results in an increase of FCC atoms [25].

In contrast to the decreasing FCC atoms in single crystalline $\mathrm{Cu}$ NWs and twinned ones with large TBS, Figure 3 shows that the number of FCC atoms in NW1 with the
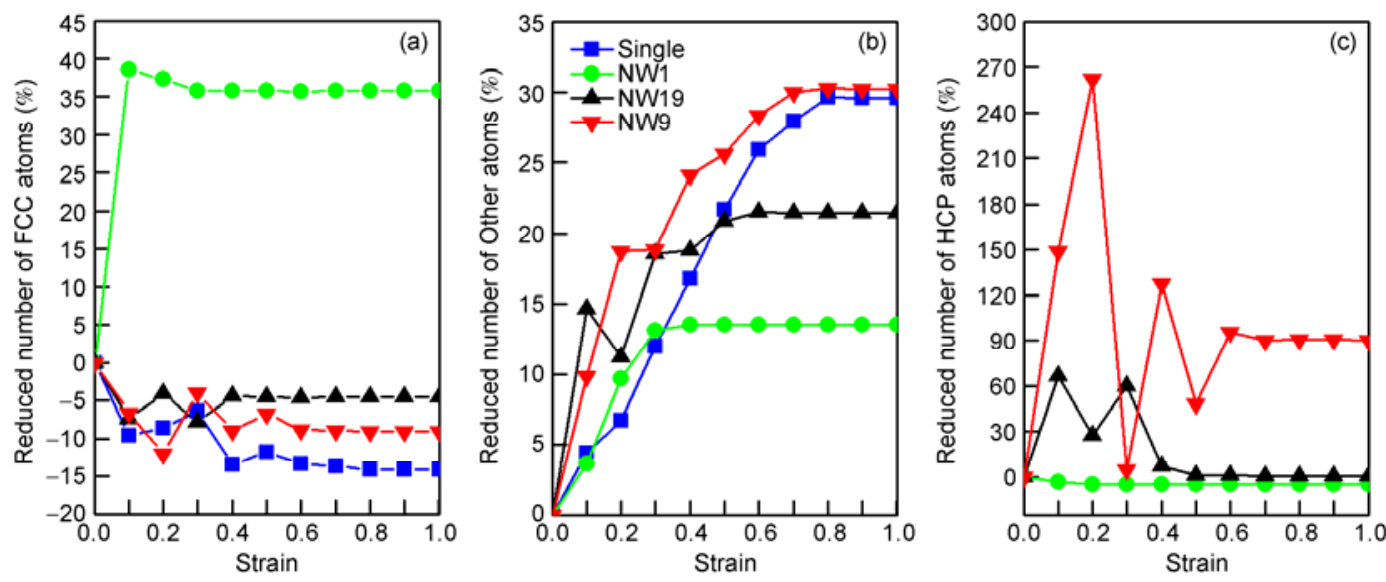

Figure 3 Evolutions of atoms in different lattice structures during the tension process. (a) Reduced number of FCC atoms; (b) reduced number of Other atoms; (c) reduced number of HCP atoms. The reduced number is calculated by subtracting the base number before deformation using the measured number. 
smallest TBS increases during plastic deformation, accompanied with simultaneously increase in the Other atoms. Since the increment of Other atoms in the NW1 is the smallest and there is no reduction in FCC atoms, it suggests that the dislocation density in NW1 is minor and dislocation activity cannot be the dominant deformation mechanism. To reveal the deformation mechanisms of NW1, Figure 3(c) further presents the evolutions of HCP atoms in twinned NWs upon strain. It is seen that during plastic deformation the number of HCP atoms in the twinned NWs with large TBS increases with strong fluctuations, which are resulted from the dissociation of lattice partial dislocations into mechanical TBs. However, the number of HCP atoms in NW1 decreases slightly. The decrease of HCP atoms can be caused by detwinning, i.e., disappearance of TBs, in addition to the formation of mechanical TBs. However, deformation twinning cannot be the dominant plastic deformation mode of NW1 with TBS of $0.63 \mathrm{~nm}$, which is the minimum period of stacking sequence of $\mathrm{ABC}$ in [111] crystallographic orientation of $\mathrm{Cu}$ lattice. Thus, it reveals that the plastic deformation of NW1 is dominated by detwinning.

Figure 4 presents cross-sectional views of $\mathrm{Cu}$ NWs at their fracture points, which shows that there is a neck region formed accompanied with failure in each NWs. Furthermore, the length of neck region is larger in NWs with larger fracture strain. Figure 4 also demonstrates that the failure position of NWs varies significantly upon internal microstructure. Previous atomistic simulations suggested that the failure position of NWs under nano-stretching strongly depends on the NWs length, and follows a statistical distribution $[13,26]$. Figure 4 (a) shows that there is no TB observed in the neck region of NW1, and dislocation activity in the NWs is severely restricted by TBs. Furthermore, the TBs near the neck region of NW1 undergo significant TB migration, as evidenced by their increased TBS. The occurrence of significant TB migration and subsequent detwinning in NW1 provides increased space to accommodate nucleation and subsequent glide of lattice dislocations. Correspondingly, the number of FCC atoms in NW1 first increases

(a)

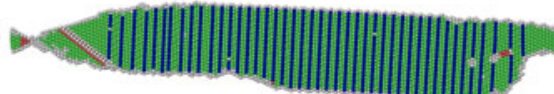

(b)

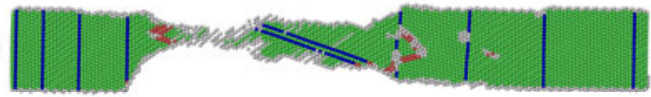

(c)

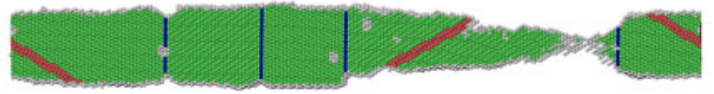

(d)

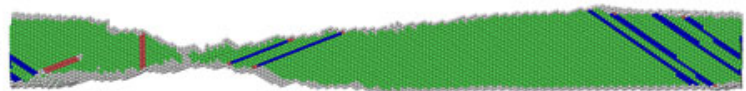

Figure 4 Cross-sectional views of $\mathrm{Cu}$ NWs at fracture strain. (a) NW1; (b) NW19; (c) NW9; (d) single. Atoms are colored according to CNA values. dramatically caused by detwinning, followed by minor decrease due to the emergence of dislocation activity, as shown in Figure 3(a). In contrast to glide of lattice partial dislocations parallel to twin planes in bulk nanotwinned $\mathrm{Cu}$ with small TBS, Figure 4 shows that lattice dislocations are mainly inclined to TBs for all the twinned $\mathrm{Cu}$ NWs. Figure 4(a) and (b) shows that lattice partials nucleate from TBs that lose their coherency due to dislocation-TB interaction. However, TB migration is not pronounced in twinned $\mathrm{Cu}$ NWs with large TBS, the plastic deformation of which is dominated by dislocation activity. Figures 3 and 4 jointly demonstrate that dislocation activity has strong dependence on twin lamellae thickness: the larger the TBS, the higher the dislocation density, and the higher the tensile ductility.

To probe the mechanism of dominant detwinning in NW1 with the thinnest twin lamellae thickness, the instantaneous deformation structures prior to the occurrence of fracture are analyzed. Figure 5(a) shows that the plasticity of NW1 is governed by the nucleation of twinning partials and resulting TBs losing coherency, accompanied with the nucleation and subsequent glide of lattice partials parallel to twin planes. The glide of lattice partials on twin planes leads to shrinking of TBs and formation of three HCP-coordinated layers. Figure 5(b) shows the widening of twin lamellae thickness in the neck region due to TB migration, and complete annihilation of lattice partials is also observed. Upon further loading, TBS is significantly increased to provide additional space to accommodate dislocation activity. Figure 5(c) shows that a leading partial nucleates from the surface of neck region and intersects with TB. Consequently, TB migration and detwinning triggered by the dislocationTB interaction occur again. The thickness of twin lamellae containing leading and trailing partials shown in Figure 5(d) is $3.75 \mathrm{~nm}$, increased by a factor of 6 in comparison with the original value of $0.63 \mathrm{~nm}$. Figure 5 reveals that TB migration and subsequent detwinning are originated from the nucleation and subsequent glide of lattice partial dislocations parallel to the twin planes, which is in good agreement with recent experimental studies and atomistic simulations [11,27-29].

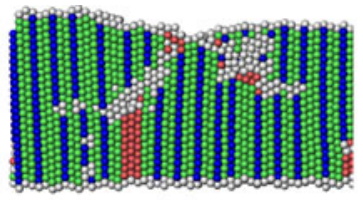

(a)

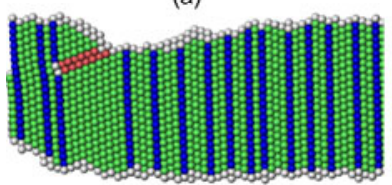

(c)

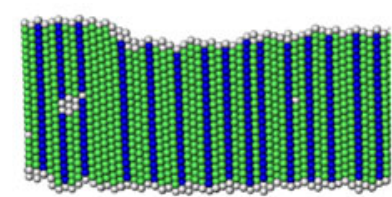

(b)

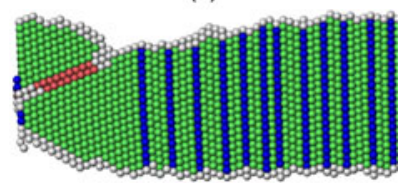

(d)
Figure 5 Instantaneous deformation structures of NW1 during detwinning process. Strain of (a) 0.084 ; (b) 0.144 ; (c) 0.16 ; (d) 0.176 . 


\section{Summary}

In summary, we perform MD simulations to investigate the uniaxial tension of twinned $\mathrm{Cu}$ NWs containing high density of parallel TBs. Simulation results reveal that the plastic deformation modes of $\mathrm{Cu}$ NWs include dislocation activity, deformation twinning, TB migration and detwinning. Furthermore, twin lamellae thickness has strong influence on the competition between individual deformation modes, which in turn affects the tensile ductility of $\mathrm{Cu}$ NWs: the smaller the TBS, the smaller the fracture strain. In particular for the smallest TBS of $0.63 \mathrm{~nm}$, the dominant detwinning mechanism leads to a significant reduction in the tensile ductility of the twinned NWs. It reveals that TB migration and subsequent detwinning originate from the nucleation and glide of lattice partial dislocations parallel to the twin planes.

This work was supported by China Postdoctoral Science Foundation (2012M511463), Heilongjiang Postdoctoral Foundation of China (LBHZ11143) and the ICAMS, Ruhr-University Bochum, Germany.

1 Lu L, Shen Y F, Chen X H, et al. Ultrahigh strength and high electrical conductivity in copper. Science, 2004, 304: 422-426

2 Anderoglu O, Misra A, Wang $\mathrm{H}$, et al. Epitaxial nanotwinned $\mathrm{Cu}$ films with high strength and high conductivity. Appl Phys Lett, 2008, 93: 083108

3 Ma E, Wang Y M, Lu Q H, et al. Strain hardening and large tensile elongation in ultrahigh-strength nano-twinned copper. Appl Phys Lett, 2004, 85: 4932-4934

4 Lu K, Lu L, Suresh S. Strengthening materials by engineering coherent internal boundaries at the nanoscale. Science, 2009, 324: 349352

5 Cao A J, Wei Y G. Atomistic simulations of the mechanical behavior of fivefold twinned nanowires. Phys Rev B, 2006, 74: 214108

6 Wu J Y, Nagao S, He J Y, et al. Role of five-fold twin boundary on the enhanced mechanical properties of fcc Fe nanowires. Nano Lett, 2011, 11: 5264-5273

7 Gao Y J, Fu Y Q, Sun W, et al. Investigation on the mechanical behavior of fivefold twinned silver nanowires. Comp Mater Sci, 2012, 55: 322-328

8 Sansoz F, Deng C. Size-dependent plasticity in twinned metal nanowires. In: Proceedings of the International Conference on Fracture (ICF) 12, 2009, Ottawa, Canada. 4-9

9 Lu Y, Song J, Huang J Y, et al. Fracture of sub-20 nm ultrathin gold nanowires. Adv Func Mater, 2011, 21: 3982-3989
10 Lu L, Chen X, Huang X, et al. Revealing the maximum strength in nanotwinned copper. Science, 2009, 323: 607-610

11 Li X Y, Wei Y J, Lu L, et al. Dislocation nucleation governed softening and maximum strength in nanotwinned metals. Nature, 2010, 464: 877-880

12 Foiles S M, Baskes M I, Daw M S. Embedded-atom-method functions for the fcc metals $\mathrm{Cu}, \mathrm{Au}, \mathrm{Ni}, \mathrm{Pd}, \mathrm{Pt}$, and their alloys. Phys Rev B, 1986, 33: 7983-7991

13 Liu Y H, Zhao J W, Wang F Y. Influence of length on shock-induced breaking behavior of copper nanowires. Phys Rev B, 2009, 80: 115417

14 Wei Y J. Anisotropic size effect in strength in coherent nanowires with tilted twins. Phys Rev B, 2011, 84: 014107

15 Honeycutt J D, Andersen H C. Molecular dynamics study of melting and freezing of small Lennard-Jones clusters. J Phys Chem, 1987, 91: 4950-4963

16 Stukowski A, Albe K. Dislocation derection algorithm for atomistic simulations. Modelling Simul Mater Sci Eng, 2010, 18: 025016

17 Plimpton S. Fast parallel algorithms for short-range molecular dynamics. J Comp Phys, 1995, 117: 1-19

18 Stukowski A. Visualization and analysis of atomistic simulation data with OVITO-the Open Visualization Tool. Modelling Simul Mater Sci Eng, 2010, 18: 015012

19 Cao A J, Wei Y G, Mao S X. Deformation mechanisms of face-centered-cubic metal nanowires with twin boundaries. Appl Phys Lett, 2007, 90: 151909

20 Deng C, Sansoz F. Effects of twin and surface facet on strain-rate sensitivity of gold nanowires at different temperatures. Phys Rev B, 2010, 81: 155430

21 Cao A J, Ma E. Sample shape and temperature strongly influence the yield strength of metallic nanopillars. Acta Mater, 2008, 56: 4816-4828

22 Gao Y J, Wang H B, Zhao J W, et al. Anisotropic and temperature effects on mechanical properties of copper nanowires under tensile loading. Comp Mater Sci, 2011, 50: 3032-3037

23 You Z S, Lu L, Lu K. Temperature effect on rolling behavior of nano-twinned copper. Scripta Mater, 2010, 62: 415-418

24 Sun J P, Fang L, Sun K, et al. Direct observation of dislocations originating from perfect twin boundaries. Scripta Mater, 2011, 65: 501-504

25 Liao X Z, Zhao Y H, Srinivasan S G, et al. Deformation twinning in nanocrystalline copper at room temperature and low strain rate. Appl Phys Lett, 2004, 84: 592-594

26 Wang D X, Zhao J W, Hu S, et al. Where, and how, does a nanowire break? Nano Lett, 2007, 7: 1208-1212

27 Wang J, Li N, Anderoglu O, et al. Detwinning mechanisms for growth twins in face-centered cubic metals. Acta Mater, 2010, 58: 2262-2270

28 Wang Y D, Liu W J, Lu L, et al. Low temperature deformation detwinning-A reverse mode of twinning. Adv Eng Mater, 2010, 12: 906-911

29 Wei Y J. The kinetics and energetic of dislocation mediated de-twinning in nano-twinned face-centered cubic metals. Mater Sci Eng A, 2011, 528: 1558-1566

Open Access This article is distributed under the terms of the Creative Commons Attribution License which permits any use, distribution, and reproduction in any medium, provided the original author(s) and source are credited. 